Published in final edited form as:

ACS Sens. 2020 February 28; 5(2): 296-302. doi:10.1021/acssensors.9b01736.

\title{
Digital Receptor Occupancy Assay in Quantifying On- and Off- Target Binding Affinities of Therapeutic Antibodies
}

\author{
Chao-Kai Chou \\ Department of Molecular and Cellular Oncology, The University of Texas MD Anderson Cancer \\ Center, Houston, Texas 77030, United States \\ Yen-Liang Liu \\ Graduate Institute of Biomedical Sciences, China Medical University, Taichung, Taiwan 40402; \\ Department of Biomedical Engineering, The University of Texas at Austin, Austin, Texas 78712, \\ United States;
}

\section{Yuan-I Chen,}

Department of Biomedical Engineering, The University of Texas at Austin, Austin, Texas 78712, United States

\section{Po-Jung Huang}

Department of Materials Science and Engineering, Texas A\&M University, College Station, Texas 77840, United States;

\author{
Pei-Hsiang Tsou, Chun-Te Chen, Heng-Huan Lee, Ying-Nai Wang, Jennifer L. Hsu, Jin-Fong \\ Lee \\ Department of Molecular and Cellular Oncology, The University of Texas MD Anderson Cancer \\ Center, Houston, Texas 77030, United States
}

\section{Thomas E. Yankeelov,}

Department of Biomedical Engineering, Department of Diagnostic Medicine, Dell Medical School, Department of Oncology, Dell Medical School, and Livestrong Cancer Institutes, The University of Texas at Austin, Austin, Texas 78712, United States;

\footnotetext{
Corresponding Authors: Jun Kameoka - Department of Materials Science and Engineering and Department of Electrical and Computer Engineering, Texas A\&M University, College Station, Texas 77840, United States; kameoka@ mail.ece.tamu.edu, HsinChih Yeh - Department of Biomedical Engineering and Texas Materials Institute, The University of Texas at Austin, Austin, Texas 78712, United States; tim.yeh@ austin.utexas.edu, Mien-Chie Hung - Department of Molecular and Cellular Oncology, The University of Texas MD Anderson Cancer Center, Houston, Texas 77030, United States; Graduate Institute of Biomedical Sciences and Center for Molecular Medicine, China Medical University, Taichung, Taiwan 40402; Cancer Biology Program, Graduate School of Biomedical Sciences, The University of Texas Health Sciences Center at Houston, Houston, Texas 77030, United States; Department of Biotechnology, Asia University, Taichung, Taiwan 41354; mhung@ mail.cmu.edu.tw.

Author Contributions

C.-K. C. conceived the project; C.-K. C., P.-J. H, and P.-H. T. performed the DRO analysis; C.-T. C. supported the tissue sample collection; C.-K. C., H.-H. L., Y.-N. W., and J.-F. L. performed cell culture and sample preparation; Y.-L. L., Y.-I. C., T. E. Y., and H.C. Y. helped the optical system and performed data analysis; Y.-I. C. conducted the experiments of flow-cytometry and fluorescence imaging. C.-K. C., Y.-L. L., H.-C. Y., J. L. H., and P.-J. H. wrote the manuscript; J. K., H.-C. Y., and M.-C. H. supervised the project. C.-K C. and Y.-L. L. contributed equally to this work.

Supporting Information

The Supporting Information is available free of charge at https://pubs.acs.org/doi/10.1021/acssensors.9b01736.

Detailed information about materials, methods, DRO optical system, microchannel fabrication, and quantification of DRO assay (PDF)

The authors declare no competing financial interest.
} 
Institute for Computational Engineering and Sciences, The University of Texas, Austin, Texas 78712, United States

Jun Kameoka,

Department of Materials Science and Engineering and Department of Electrical and Computer Engineering, Texas A\&M University, College Station, Texas 77840, United States;

Hsin-Chih Yeh, Department of Biomedical Engineering and Texas Materials Institute, The University of Texas at Austin, Austin, Texas 78712, United States;

Mien-Chie Hung

Department of Molecular and Cellular Oncology, The University of Texas MD Anderson Cancer Center, Houston, Texas 77030, United States;

Graduate Institute of Biomedical Sciences and Center for Molecular Medicine, China Medical University, Taichung, Taiwan 40402;

Cancer Biology Program, Graduate School of Biomedical Sciences, The University of Texas Health Sciences Center at Houston, Houston, Texas 77030, United States; Department of Biotechnology, Asia University, Taichung, Taiwan 41354;

\section{Abstract}

While monoclonal antibodies are the fastest-growing class of therapeutic agents, we lack a method that can directly quantify the on- and off-target binding affinities of newly developed therapeutic antibodies in crude cell lysates. As a result, some therapeutic antibody candidates could have a moderate on-target binding affinity but a high off-target binding affinity, which not only gives a reduced efficacy but triggers unwanted side effects. Here, we report a single-molecule counting method that precisely quantifies antibody-bound receptors, free receptors, and unbound antibodies in crude cell lysates, termed digital receptor occupancy assay (DRO). Compared to the traditional flow cytometry-based binding assay, DRO assay enables direct and digital quantification of the three molecular species in solution without the additional antibodies for competitive binding. When characterizing the therapeutic antibody, cetuximab, using DRO assay, we found the ontarget binding ratio to be $65 \%$ and the binding constant $\left(K_{\mathrm{d}}\right)$ to be $2.4 \mathrm{nM}$, while the off-target binding causes the binding constant to decrease by $0.3 \mathrm{nM}$. Other than cultured cells, the DRO assay can be performed on tumor mouse xenograft models. Thus, DRO is a simple and highly quantitative method for cell-based antibody binding analysis which can be broadly applied to screen and validate new therapeutic antibodies.

\section{Graphical Abstract}



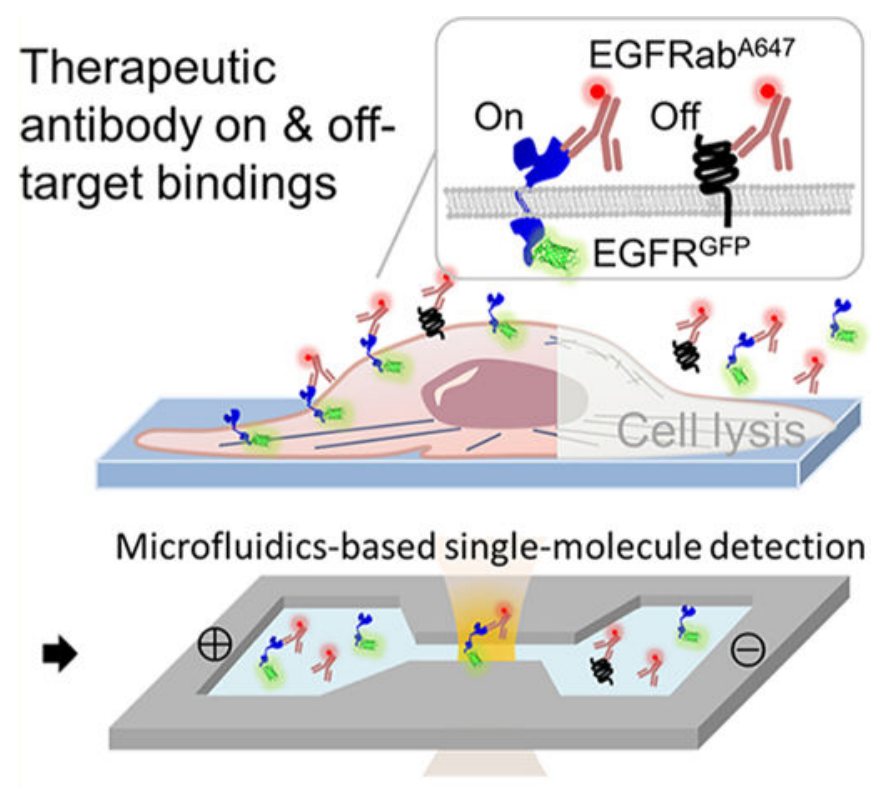

Two-color fluorescence coincidence analysis

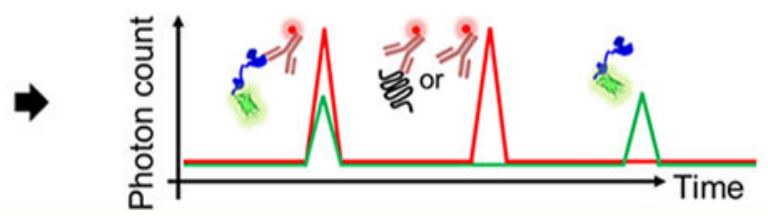

\section{Keywords}

antibody binding affinity; receptor occupancy; on-target binding; on-target quantification; singlemolecule detection; microjluidics

Achieving a high on-target binding affinity but a low off-target affinity is always at the center of all therapeutic monoclonal antibody development. ${ }^{1}$ With a vast amount of new monoclonal antibodies produced every year, ${ }^{2}$ it is critically important to rapidly screen these antibodies and identify the ones with the highest on-target binding affinity and specificity. ${ }^{3}$

Currently, several methods are routinely used for characterizing the antibody binding metrics in the cell-free condition, including enzyme-linked immunosorbent assay (ELISA), ${ }^{4,5}$ surface plasmon resonance (SPR), ${ }^{6,7}$ and biolayer interferometry. ${ }^{8}$ While these cell-free measurements are simple and reliable, the results may not reflect the true binding affinities in the cellular environment. ${ }^{9}$ To bypass this limitation and evaluate the antibody's pharmacodynamics, flow cytometry-based receptor occupancy assay is often used to quantify the antibody-bound receptors and the unbound receptors in cells, revealing the binding status during the treatment. ${ }^{10-12}$ However, flow cytometry analysis has two major problems. First, the number of unbound receptors is usually estimated from the amount of a competitive antibody that binds to the receptors previously unoccupied by the therapeutic antibody. Not only is the application of competitive antibody a cumbersome labeling process, but it is also difficult to have a "perfect" competitive antibody that binds to the same epitope on the target and reaches identical binding strength (as compared with the 
therapeutic antibody). ${ }^{11}$ In addition, the assessment of unbound receptors can be obstructed by the interfering factors that neutralize competitive antibody. ${ }^{11}$ Second, flow cytometry analysis employs additional antibodies to label the receptors and the therapeutic antibodies separately. The off-target binding affinities of these additional labeling antibodies can bias the estimate..$^{3,13,14}$ There is, therefore, an urgent need to create a new quantification method that not only enables "digital" on- and off-target binding quantification in cells but bypasses the use of any additional antibodies for measurement. Microfluidics-based single-molecule detection provides a direct method to observe biomolecule interactions and count molecular species one by one. For instance, Yeh et al. developed a two-color fluorescence coincidence detection method in a microcapillary and digitally counted wild-type and mutant DNA. ${ }^{15}$ Wadhwa et al. invented a microfluidic immunoassay for distinguishing bacterium-caused Johne's disease. ${ }^{16}$ Recently, we also developed a microchannel-based flow-proteometric technique that enables digital quantification of target proteins and related complexes from fixed biological samples. ${ }^{17}$ In our flow-proteometric analysis, each photon burst represents a fluorescently labeled molecule or molecular complex which flows through the detection volume in the microchannel (Figure 1A). As the microchannel width $(2 \mu \mathrm{m})$ is only 2.5 -fold larger than radial dimension of the detection volume $(0.8 \mu \mathrm{m})$, and the channel height (500 $\mathrm{nm})$ is smaller than the axial dimension of the detection volume $(3 \mu \mathrm{m})$ (Figure 1B), our mass detection efficiency can be as high as $31 \%$. Here, we extended this flow-proteometric assay to a "cell-based" assay that enables direct single-molecule quantification of therapeutic antibodies binding to the target receptors in cells, which we term digital receptor occupancy assay (DRO). It is known that epidermal growth factor receptor (EGFR) deregulation causes cancer progression, and anti-EGFR antibody (cetuximab, EGFRab hereinafter ${ }^{18}$ has been used to treat colorectal cancer, ${ }^{19}$ non-small cell lung cancer, ${ }^{20}$ and head and neck cancer. ${ }^{21}$ Using EGFRab as an example, here we digitally count the numbers of EGFRab, EGFR, and EGFRab-EGFR complexes in DRO assay after treating cancer cells with EGFRab (Figure 1A). Without the need to use competitive and labeling antibodies, DRO assay provides bias-free on- and off-target binding affinities of EGFRab in cancer cells. Not only is it a highly quantitative cell-based assay, but DRO can also complete antibody binding measurement in a few hours using a minute amount of sample $\left(\sim 10^{4}\right.$ cells $)$.

In a typical DRO assay, cells expressing the target receptors fused with the green fluorescent protein (i.e., EGFR ${ }^{\mathrm{GFP}}$ ) were treated with Alexa Fluor 647 (A647)-conjugated therapeutic antibody (i.e., EGFRab ${ }^{\mathrm{A} 647}$ ). After treatment, the unbound antibodies were removed by phosphate-buffered saline (PBS) rinse and the cells were sequentially lysed and diluted for microfluidic measurements. In this protocol, only the antibodies ever associated with cells can be detected in the DRO assay. In the EGFRab binding experiment, we first generated a stable cell line that expresses EGFR ${ }^{\mathrm{GFP}}$ in the EGFR-null Chinese hamster ovary cells (CHO-EGFR ${ }^{\mathrm{GFP}}$ ) and treated the cells with A647-conjugated EGFRab (EGFRab ${ }^{\mathrm{A} 647}$ ) under the designated condition, followed by a collection of the crude cell lysate for DRO analysis. The coincident detection method is able to identify EGFR ${ }^{\mathrm{GFP}}, \mathrm{EGFRab}^{\mathrm{A} 647}$, and the bound complex. A green photon burst represented EGFR ${ }^{\text {GFP }}$ without EGFRab ${ }^{\mathrm{A} 6 i 7}$ binding (Figure 1C, top), whereas a red photon burst represented EGFRab ${ }^{\mathrm{AS} 47}$ without $\mathrm{EQFR}^{\mathrm{GFP}}$ binding (Figure 1C, middle). Coincident green and red photon bursts thus indicated an EGFRab ${ }^{\mathrm{A} 647}$-EGFR ${ }^{\mathrm{GFP}}$ binding complex (Figure 1C, bottom). As the sample 
flowed through the detection volume, individual photon bursts were seen in the two fluorescence time traces (Figure 1D). EGFR occupancy ratio and dissociation constant $\left(K_{\mathrm{d}}\right)$ of EGFRab-EGFR complex could then be computed from the photon burst counting results. The binding fractions (BF) based on EGFR ${ }^{\text {GFP }}$ or EGFRab ${ }^{\mathrm{Al} 547}$ were derived through the DRO assay to reveal in an unbiased manner the interaction between EGFR and anti-EGFR antibody (Figure 1E). The EGFR ${ }^{\mathrm{GFP}} \mathrm{BF}$ indicates the portion of EGFR ${ }^{\mathrm{GFP}}$ recognized by EGFRab $^{\mathrm{A} 647}$, and the EGFRab ${ }^{\mathrm{A} 647}$ BF represents the percentile of EGFRab ${ }^{\mathrm{A} 647}$ binding to $\mathrm{EGFR}^{\mathrm{GFP}}$. Please see Supporting Information for the detailed description.

To evaluate the treatment dynamics, we measured EGFRab ${ }^{\mathrm{A} 647}$-EGFR ${ }^{\mathrm{GFP}}$ binding affinity in $\mathrm{CHO}$ cells treated with $20 \mathrm{nM}$ EGFRab ${ }^{\mathrm{A} 647}$ for 5 to $60 \mathrm{~min}$. From the $5 \mathrm{~min}$ treatment sample, 1,861 EGFRab ${ }^{4647}$ events per 5,000 EGFR ${ }^{\text {GFP }}$ events were detected (on average, Figure 2A). Out of these 1,861 EGFRab ${ }^{\mathrm{A} 647}$ events, 1,038 were EGFRab ${ }^{\mathrm{A} 647}$ - EGFR GFP complex events. Extending the treatment time increased the number of EGFRab ${ }^{\mathrm{A} 647}$ events, but the number peaked at $30 \mathrm{~min}$. The fraction of EGFRab ${ }^{\mathrm{A} 647}$ bound to EGFR ${ }^{\mathrm{GFP}}$ was nearly $60 \%$ at $5 \mathrm{~min}$, but reduced to $40 \%$ at $60 \mathrm{~min}$ (Figure 2B). Interestingly, the fraction of $\mathrm{EGFR}^{\mathrm{GFP}}$ involved in EGFRab ${ }^{\mathrm{A} 647}$ binding remained the same during the 60 min treatment. These results reflected the intriguing dynamic process of EGFRab ${ }^{\mathrm{A} 647}$ in targeting $\mathrm{EGFR}^{\mathrm{GFP}}$.

As the highest EGFRab ${ }^{\mathrm{A} 647}$-EGFR ${ }^{\mathrm{GFP}}$ binding ratio was achieved within the first 5 min of treatment, we set this 5 min time point as the basis for generating the EGFRab ${ }^{\mathrm{A} 647}$ EGFR $^{\text {GFP }}$ binding curve. The Scatchard plots were used to determine the dissociation constants $\left(K_{\mathrm{d}}\right)$ and the maximum number of targetable EGFRs $\left(B_{\max }\right)$. Please see Supporting Information for a detailed description of the Scatchard equation. When plotting the binding saturation curve based on the EGFRab ${ }^{\mathrm{A} 647}$ event numbers (which represent the EGFRab $^{\mathrm{A} 647}$ ever associated with cells), we found the $K_{\mathrm{d}}$ to be $2.126 \mathrm{nM}$ (Figure 2C), similar to the value reported previously. ${ }^{21}$ To compare the DRO assay with other conventional receptor occupancy assay, we conducted flow cytometry-based receptor occupancy assay, and the measured $K_{\mathrm{d}}$ was $0.68 \mathrm{nM}$ (Figure S1). Kang's group has reported that the binding affinity of Cetuximab to EGFR on the cell surface was $5 \mathrm{nM}$ and $0.38 \mathrm{nM}$ based on SPR and flow cytometry-based occupancy assay, respectively. ${ }^{22}$ Besides, Tao's group reported that the binding affinity of an anti-EGFR monoclonal antibody assessed by with SPR was between 0.1 and $0.7 \mathrm{nM} .^{23}$ The discrepancy might be attributed to different instruments, cell samples, and antibodies. In addition, the discrepancies can be due to the differences in antigen presentation and cell surface receptor density, which were reported by Chiu's group. ${ }^{24}$ However, we thought that our DRO assay is comparable to other occupancy assays because the difference of dissociation constants was within 1 order of magnitude.

We found that the $K_{\mathrm{d}}$ slightly increased by $0.298 \mathrm{nM}$ to become $2.424 \mathrm{nM}$ (Figure 2D) when plotting the curve based on the EGFRab ${ }^{\mathrm{A} 647}-\mathrm{EGFR}^{\mathrm{GFP}}$ event numbers (which represent the actual receptor occupancy). This increase in $K_{\mathrm{d}}$ implied off-target binding of EGFRab to other molecules in cells. The capability to dissect on- and off-target bindings is advantageous over conventional receptor occupancy assays, such as flow cytometry, SPR We next calculated the fractions of EGFRab ${ }^{\mathrm{A} 647}$ and EGFR GFP involved in EGFRab ${ }^{\mathrm{A} 647}$ EGFR $^{\text {GFP }}$ binding. Based on the total EGFR ${ }^{\text {GFP }}$ numbers, the $K_{\mathrm{d}}$ of EGFRab ${ }^{\mathrm{A} 647}$ 
EGFR $^{\mathrm{GPP}}$ complexes was $2.436 \mathrm{nM}$ (Figure 2E), which was identical to the $K_{\mathrm{d}}$ calculated from the number of binding events (Figure 2D). Interestingly, the fractions of the complex in the EGFRab ${ }^{\mathrm{A} 647}$ events were all around 65\%, regardless of the EGPRab ${ }^{\mathrm{A} 647}$ treatment concentration (Figure 2F). This result suggested that $65 \%$ of EGFRab ${ }^{\mathrm{A} 647}$ remained bound to EGFR ${ }^{\mathrm{GFP}}$ even after PBS wash (mimicking posttreatment in patients), and the bound antibodies would consistently provide the blockade effect (Figure 2G). As a result, we hypothesize that the EGFRab ${ }^{\mathrm{A} 647}$ binding fraction could serve as an indicator to evaluate the binding efficacy of therapeutic antibodies, which could be applied for antibody selection and treatment dosage optimization. In summary, our results demonstrated that the DRO assay is able to accurately access the antibody's ability in binding targets and maintaining the occupancy after treatment, which leads to the blockade of EGFR signaling.

As EGF-stimulated EGFR endocytosis can prevent EGFR from binding the therapeutic antibody, we also evaluated the influence of EGF on EGFRab ${ }^{\mathrm{A} 647}$-EGFR ${ }^{\mathrm{GFP}}$ binding. CHO-EGFR ${ }^{\text {GFP }}$ cells were pretreated with or without $50 \mathrm{ng} / \mathrm{mL}$ EGF for $30 \mathrm{~min}$, then followed by $15 \mathrm{nM}$ EGFRab ${ }^{\mathrm{A} 647}$ treatment for $5 \mathrm{~min}$. As expected, after EGF treatment, the fraction of EGFR ${ }^{\mathrm{GFP}}$ involved in EGFRab ${ }^{\mathrm{A} 647}$ binding was significantly decreased (Figure $2 \mathrm{H}$ ). To confirm that EGF-induced EGFR endocytosis, as reported previously, ${ }^{25}$ was indeed responsible for the reduced surface EGFR for EGFRab ${ }^{\mathrm{A} 647}$ targeting, we quantified the EGF-stimulated EGFR internalization using fluorescence imaging and also suppressed endocytosis by lowering the treatment temperature to $4{ }^{\circ} \mathrm{C}$. The surface EGFR significantly decreased after EGF stimulation (Figure S2), and the DRO result did show that EGF had no influence on the on-target binding at $4{ }^{\circ} \mathrm{C}$ (Figure $2 \mathrm{H}$ ). We also found the fraction of EGFRab $^{\mathrm{A} 647}$ bound with EGFR ${ }^{\mathrm{GFP}}$ was slightly increased upon the EGF treatment (Figure 2I); however, no obvious difference was seen in the fraction of EGFRab ${ }^{\mathrm{A} 647}$ binding when endocytosis was suppressed (Figure 2I). These results indicated that environmental alterations could affect the number of receptors available for targeting.

To demonstrate the use of DRO in receptor occupancy analysis in vivo, we generated a xenograft model of EGFR ${ }^{\mathrm{GFP}}$-expressing colorectal cancer as previously described ${ }^{26}$ (Figure $3 \mathrm{~A})$. Mice with subcutaneous tumors arising from colorectal cancer cells stably expressing EGFR $^{\text {GFP }}$ (GEO-EGFR ${ }^{\text {GFP }}$ cells with low-level endogenous EGFR expression) received intravenous injections of EGFRab ${ }^{\mathrm{A} 647}$ or isotype control $\mathrm{IgG}^{\mathrm{A} 647}$, and small pieces of tumor tissue were collected at predefined time points ( 5 and 30 min after treatment). The tumor tissue samples were then degraded using tissue homogenizer with lysis buffer, centrifuged to remove large debris, and analyzed with DRO assay. Five minutes after injection, about $28 \%$ of the EGFR ${ }^{\mathrm{GFP}}$ events were EGFRab ${ }^{\mathrm{A} 647}$-EGFR $^{\mathrm{GFP}}$ binding events. Thirty minutes after injection, the fraction slightly increased to 33\% (Figure 3B). The xenograft model showed EGFRab ${ }^{\mathrm{A} 647}$ binding dynamics similar to that of the cell culture model-a large portion of the available EGER ${ }^{\mathrm{GFP}}$ was targeted by EGFRab ${ }^{\mathrm{A} 647}$ in the first $5 \mathrm{~min}$. Thus, we demonstrate that DRO assay is capable of directly quantifying antibody-receptor binding in both the crude cell lysate and tissue lysate.

As monoclonal antibody-based target therapy, including immune checkpoint therapy, has gained tremendous interest in cancer treatment in recent years, ${ }^{27}$ here we developed a digital receptor occupancy (DRO) assay that can rapidly evaluate therapeutic antibody candidates 
and provide highly quantitative, bias-free, and cell-based target binding characterization results based on a minute amount of sample. Not only identifying the on- and off-target binding affinity of therapeutic antibody, ${ }^{28}$ our DRO assay also provides an accurate estimate of target molecules expressed in cancer cells, which is critical in effective antibody treatment. ${ }^{29,30}$ Whereas the surface-based methods, such as ELISA, ${ }^{4,5} \mathrm{SPR},{ }^{6,7}$ and polarization-modulated oblique-incidence reflectivity difference (OI-RD), ${ }^{31}$ are still the dominant methods for $K_{\mathrm{d}}$ measurements, they require the antibodies to be captured by the surface-bound targets on a chemically functionalized microplate (with ELISA), metal-coated glass slide (with SPR), or glass slide (with OI-RD), which is not a physiologically relevant condition. The unknown surface effects may have altered the binding affinity and specificity of the antibody. In contrast, our DRO is completely a cell- and solution-based assay; in other words, our method preserves the molecule's conformational, rotational, and binding degrees of freedom, and eliminates any binding interference from the surface. Whereas the traditional flow cytometry-based receptor occupancy assay ${ }^{10}$ is also a cell- based, physiologically relevant method, it requires all target receptors in cells to be labeled with labeling antibodies. Similarly, free receptors have to be labeled with competitive antibodies, and therapeutic antibodies are labeled with secondary antibodies. In such an indirect binding measurement method, any interfering factors that prevent labeling and competitive antibodies from the binding target and therapeutic antibody can skew the binding measurement results. As we directly fuse the target receptor with a fluorescence protein and label the therapeutic antibody with an organic dye, our method bypasses any artifacts resulting from the use of competitive and labeling antibodies. As shown in Figure 2, DRO assay can easily identify and precisely quantify antibody-bound receptors, free receptors, and unbound therapeutic antibodies with a coincident detection method. From the singlemolecule counts, the occupation ratio and on-target binding dissociation constant can be calculated. In addition, DRO also quantifies unbound therapeutic antibodies remaining in cells after wash. These unbound antibodies consist of both antibodies that bind other molecules (off-target binding) and antibodies dissociated from target receptors after treatment (Figure 2G). Simply using the number of unbound antibodies to represent the offtarget binding can lead to an overestimated result. A better strategy to determine the offtarget binding is to compare the dissociation constants $K_{\mathrm{d}}$ between antibody to the cell (Figure 2C) and antibody to the receptor (Figure 2D), where the difference $(0.298 \mathrm{nM})$ is likely due to the off-target binding.

There is still space for improvement of the DRO assay. The Cetuximab is bivalent and can participate in avidity, and our current model assumes the binding of EGFR-EGFRab is monovalent. Therefore, the antibody binding affinity would be affected by antibody multivalency and avidity ${ }^{32}$ As a regime of single-molecule detection, the DRO has the potential to recognize the number of Alexa 647 on single antibodies and the number of EGFRs captured by single antibodies using advanced single-molecule techniques. ${ }^{33}$ However, more sophisticated instrumentation or analysis algorithms need to be implemented.

The binding of therapeutic antibodies to target receptors is also greatly affected by environmental factors, which may reduce the therapeutic efficacy. ${ }^{34}$ EGF stimulation not only activates the EGFR signaling cascade for cancer progression but also helps EGFR 
escape from the therapeutic antibody targeting through endocytosis. ${ }^{25}$ Therefore, strategies such as blocking EGF or suppressing EGFR endocytosis can be combined with the EGFRab treatment to increase the number of EGFR that can be effectively targeted. In summary, our platform bridges the gap between indirect and direct observation of receptor occupancy and in vitro and in vivo analysis of antibody binding dynamics, which will provide the needed support for therapeutic antibody development.

\section{Supplementary Material}

Refer to Web version on PubMed Central for supplementary material.

\section{ACKNOWLEDGMENTS}

This work was funded in part by the following: National Institutes of Health (CCSG P30 CA 016672) to the shRNA and ORFeome Core and the Clinical Trials Support Resource, R01 CA211615, R01 A1116722, and U01 CA201777; Cancer Prevention \& Research Institutes of Texas (RP160710 to M.-C. H. and RR160005 to T. E. Y.); Breast Cancer Research Foundation (BCRF-17-069); National Breast Cancer Foundation, Inc.; Patel Memorial Breast Cancer Endowment Fund; The University of Texas MD Anderson-China Medical University and Hospital Sister Institution Fund (to M.-C. H.); T32 Training Grant in Cancer Biology (5T32CA186892 to H.-H. L.); Ministry of Health and Welfare, China Medical University Hospital Cancer Research Center of Excellence (MOHW107TDU-B-212-112015 and MOHW107-TDU-B-212-114024); and Center for Biological Pathways; H.-C. Y. acknowledges the support of this work by Texas 4000, the Robert A. Welch Foundation (F-1833), National Institutes of Health (GM129617), and National Science Foundation (1611451). Y.-L. L. is a recipient of the YingTsai Young Scholar Award of China Medical University (CMU108-YTY-01) and also a recipient of Young Scholar Fellowship Program from the Ministry of Science and Technology (MOST) in Taiwan (MOST 108-2636E-039-001). We thank Drs. Shang-Wei Tsai and Jin-Chern Chiou at National Chiao Tung University in Taiwan for supporting the microchannel fabrication, Drs. Shih-Chu Liao and Beniamino Barbieri at ISS Inc. for technical support, Mr. Joseph A. Munch at the Department of Scientific Publications at MD Anderson for providing editing support, and Dr. Chi Zhao and Mr. Yin-Jui Chang at University of Texas at Austin for their help with flow cytometry experiments. T. E. Y. is a CPRIT Scholar in Cancer Research.

\section{REFERENCES}

(1). Hanack K; Messerschmidt K; Listek M Antibodies and Selection of Monoclonal Antibodies. Adv. Exp. Med. Biol 2016, 917, 11-22. [PubMed: 27236550]

(2). Buss NA; Henderson SJ; McFarlane M; Shenton JM; de Haan L Monoclonal antibody therapeutics: history and future. Curr. Opin. Pharmacol 2012, 12 (5), 615-22. [PubMed: 22920732]

(3). Baker M Reproducibility crisis: Blame it on the antibodies. Nature 2015, 521 (7552), 274-6. [PubMed: 25993940]

(4). Friguet B; Chaffotte AF; Djavadi-Ohaniance L; Goldberg ME Measurements of the true affinity constant in solution of antigen-antibody complexes by enzyme-linked immunosorbent assay. J. Immunol. Methods 1985, 77 (2), 305-19. [PubMed: 3981007]

(5). Patton A; Mullenix MC; Swanson SJ; Koren E An acid dissociation bridging ELISA for detection of antibodies directed against therapeutic proteins in the presence of antigen. J. Immunol. Methods 2005, 304 (1-2), 189-195. [PubMed: 16107258]

(6). Hahnefeld C; Drewianka S; Herberg FW Determination of kinetic data using surface plasmon resonance biosensors. Methods Mol. Med 2004, 94, 299-320. [PubMed: 14959837]

(7). Katsamba PS; Navratilova I; Calderon-Cacia M; Fan L; Thornton FL; Zhu M; Bos TV; Forte C; Friend D; Laird-Offringa I; et al. Kinetic analysis of a high-affinity antibody/antigen interaction performed by multiple Biacore users. Anal. Biochem 2006, 352 (2), 208-221. [PubMed: 16564019]

(8). Shah NB; Duncan TM Bio-layer interferometry for measuring kinetics of protein-protein interactions and allosteric ligand effects. J. Visualized Exp 2014, 84, No. e51383. 
(9). Meibohm B; Derendorf H Pharmacokinetic/pharmacodynamic studies in drug product development. J. Pharm. Sci 2002, 91(1), 18-31. [PubMed: 11782894]

(10). Litwin V; Green C; Stewart JJ Receptor occupancy by flow cytometry. Cytometry, Part B 2016, $90(2), 108-9$.

(11). Stewart JJ; Green CL; Jones N; Liang M; Xu Y; Wilkins DE; Moulard M; Czechowska EL; Lanham D; McCloskey TW; et al. Role of receptor occupancy assays by flow cytometry in drug development. Cytometry, Part B 2016, 90 (2), 110-116.

(12). Green CL; Stewart JJ; Högerkoip CM; Lackey A; Jones N; Liang M; Xu Y; Ferbas J; Moulard M; Czechowska K; et al. Recommendations for the development and validation of flow cytometrybased receptor occupancy assays. Cytometry, Part B 2016, 90 (2), 141-149.

(13). Rudmann DG On-target and off-target-based toxicologic effects. Toxicol. Pathol 2013, 41 (2), 310-4. [PubMed: 23085982]

(14). Klinger M A role for macromolecular crowding in off-target binding of therapeutic antibodies. Protein Eng., Des. Set 2017, 30 (7), 489-494.

(15). Yeh H-C; Ho Y-P; Shih I-M; Wang T-H Homogeneous point mutation detection by quantum dotmediated two-color fluorescence coincidence analysis. Nucleic Acids Res. 2006, 34 (5), e35-e35. [PubMed: 16517937]

(16). Wadhwa A; Foote RS; Shaw RW; Eda S Bead-based microfluidic immunoassay for diagnosis of Johne's disease. J. Immunol. Methods 2012, 382 (1-2), 196-202. [PubMed: 22705087]

(17). Chou CK; Lee HH; Tsou PH; Chen CT; Hsu JM; Yamaguchi H; Wang YN; Lee HJ; Hsu JL; Lee JF; Kameoka J; Hung MC mMAPS: A Flow-Proteometric Technique to Analyze Protein-Protein Interactions in Individual Signaling Complexes. Sci. Signaling 2014, 7 (315), rsl.

(18). Harding J; Burtness B An epidermal growth factor receptor chimeric human-murine monoclonal antibody. Drugs Today (Bare) 2005, 41, 107-127.

(19). Hutchinson RA; Adams RA; McArt DG; Salto-Tellez M; Jasani B; Hamilton PW Epidermal growth factor receptor immunohistochemistry: new opportunities in metastatic colorectal cancer. J. Transl. Med 2015, 13, 217. [PubMed: 26149458]

(20). Govindan R Cetuximab in advanced non-small cell lung cancer. Clin. Cancer Res 2004, 10, 4241s-4244s. [PubMed: 15217966]

(21). Kim ES; Vokes EE; Kies MS Cetuximab in cancers of the lung and head \& neck Semin. Oncol 2004, 31, 61-7. [PubMed: 14981582]

(22). Patel D; Lahiji A; Patel S; Franklin M; Jimenez X; Hicklin DJ; Kang X Monoclonal antibody cetuximab binds to and down-regulates constitutively activated epidermal growth factor receptor vIII on the cell surface. Anticancer Res. 2007, 27 (5A), 3355-3366. [PubMed: 17970081]

(23). Zhang F; Wang S; Yin L; Yang Y; Guan Y; Wang W; Xu H; Tao N Quantification of epidermal growth factor receptor expression level and binding kinetics on cell surfaces by surface plasmon resonance imaging. Anal. Chem 2015, 87 (19), 9960-9965. [PubMed: 26368334]

(24). Jarantow SW; Bushey BS; Pardinas JR; Boakye K; Lacy ER; Sanders R; Sepulveda MA; Moores SL; Chiu ML Impact of cell-surface antigen expression on target engagement and function of an epidermal growth factor receptor× c-MET bispecific antibody. J. Biol. Chem 2015, 290 (41), 24689-24704. [PubMed: 26260789]

(25). Lee HH; Wang YN; Hung MC Non-canonical signaling mode of the epidermal growth factor receptor family. Am. J. Cancer Res 2015, 5 (10), 2944-58. [PubMed: 26693051]

(26). Liao HW; Hsu JM; Xia W; Wang HL; Wang YN; Chang WC; Arold ST; Chou CK; Tsou PH; Yamaguchi H; Fang YF; Lee HJ; Lee HH; Tai SK; Yang MH; Morelli MP; Sen M; Ladbury JE; Chen CH; Grandis JR; Kopetz S; Hung MC PRMTl-mediated methylation of the EGF receptor regulates signaling and cetuximab response. J. Clin. Invest 2015, 125 (12), 4529-43. [PubMed: 26571401]

(27). Weiner LM; Surana R; Wang S Monoclonal antibodies: versatile platforms for cancer immunotherapy. Nat. Rev. Immunol 2010, 10 (5), 317. [PubMed: 20414205]

(28). Weiner LM; Murray JC; Shuptrine CW Antibody-based immunotherapy of cancer. Cell 2012, 148 (6), 1081-1084. [PubMed: 22424219]

(29). Buter J; Giaccone G EGFR inhibitors in lung cancer. Oncology (Williston Park) 2005, 19 (13), 1707. [PubMed: 16425523] 
(30). Engel RH; Kaklamani VG HER2-positive breast cancer. Drugs 2007, 67 (9), 1329-1341. [PubMed: 17547474]

(31). Lofgren JA; Dhandapani S; Pennucci JJ; Abbott CM; Mytych DT; Kallyaperumal A; Swanson SJ; Mullenix MC Comparing ELISA and surface plasmon resonance for assessing clinical immunogenicity of panltumumab.J. Immunol 2007, 178 (11), 7467-7472. [PubMed: 17513798]

(32). Rudnick SI; Adams GP Affinity and avidity in antibody-based tumor targeting. Cancer Biother.Radiopharm 2009, 24 (2), 155-161. [PubMed: 19409036]

(33). Liu C; Liu YL; Perillo EP; Dunn AK; Yeh HC Single-Molecule Tracking and Its Application in Biomolecular Binding Detection. IEEE J. Sel. Top. Quantum Electron 2016, $22,64$.

(34). Katt ME; Pla cone AL; Wong AD; Xu ZS; Searson PC In vitro tumor models: advantages, disadvantages, variables, and selecting the right platform. Front. Bioeng. Biotechnol 2016, 4, 12. [PubMed: 26904541] 
A

Antibody on \& off- target bindings
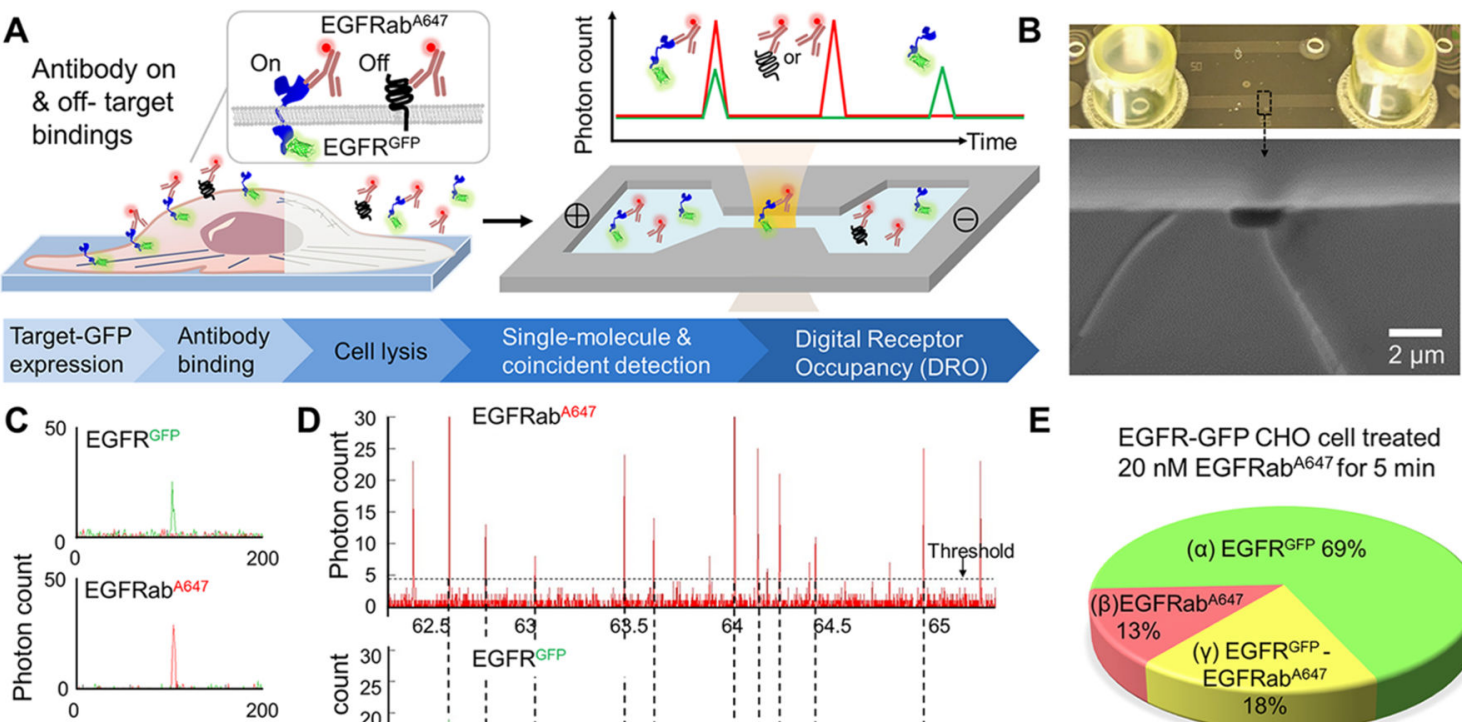

50 |EGFR ${ }^{\text {GFP-EGFRab }}{ }^{\text {A647 }}$
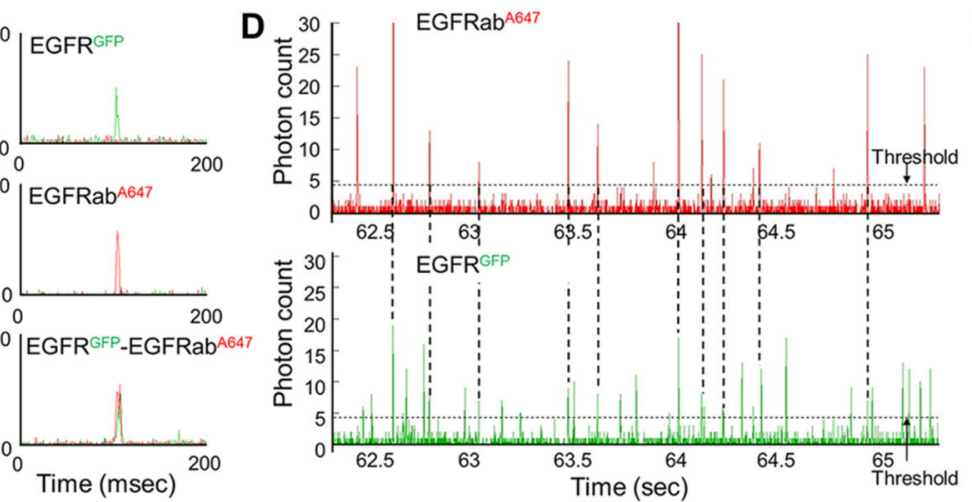

E

EGFR-GFP CHO cell treated $20 \mathrm{nM}$ EGFRab ${ }^{\mathrm{A} 647}$ for $5 \mathrm{~min}$

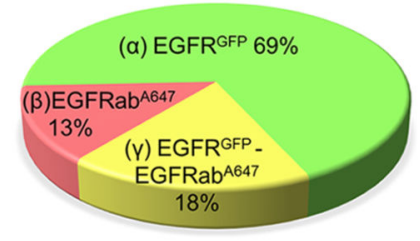

$E^{E G F R}{ }^{G F P} \mathrm{BF}=\frac{\gamma}{\alpha+\gamma} \times 100 \%=20.7 \%$

$E G F R^{a b A 647} \mathrm{BF}=\frac{\gamma}{\beta+\gamma} \times 100 \%=57.6 \%$

Figure 1.

Principle of digital receptor occupancy assay (DRO). (A) Procedure of DRO assay: first, cells were transfected with a target receptor fused with GFP (i.e., EGFR ${ }^{\mathrm{GFP}}$ ); second, the cells are treated with the A647-conjugated antibodies (i.e., A647-conjugated anti-EGFR IgG, EGFRab ${ }^{\mathrm{A} 647}$ ); third, the cells are washed with PBS to remove the unbound antibodies and lysed subsequently; fourth, the lysates are loaded into the microchannel for DRO analysis. Photon bursts from individual target proteins (green) or the antibody (red) are detected; coincident photon bursts (red and green) indicate a receptor occupied by an antibody. (B) Photo of a microchannel and the SEM image of the cross section of the microchannel. (C) Representative photon bursts showing EGFR ${ }^{\mathrm{GFP}}$ without EGFRab ${ }^{\mathrm{A} 647}$ binding (top), EGFRab ${ }^{\mathrm{A} 647}$ without EGFR ${ }^{\mathrm{GFP}}$ binding (middle), and EGFRab ${ }^{\mathrm{A} 647}$ EGFR $^{\text {GFP }}$ complex (bottom). (D) Representative time traces of photon bursts of EGFR ${ }^{\mathrm{GFP}}$ and EGFRab ${ }^{\mathrm{A} 647}$. Each red photon burst represented a single EGFRab ${ }^{\mathrm{A} 647}$ event, while each green burst represented a single EGFR ${ }^{\mathrm{GFP}}$ event. Thresholds were applied to exclude background noise and nonspecific autofluorescence signals. The dashed lines mark coincident burst events. (E) Binding fractions (BF) based on EGFR ${ }^{\text {GFP }}$ or EGFRab ${ }^{\text {A647 }}$ events were derived through the DRO assay to reveal the interaction between EGFR and anti-EGFR antibody. EGFR ${ }^{\mathrm{GFP}} \mathrm{BF}$ indicates the portion of $\mathrm{EGFR}^{\mathrm{GFP}}$ recognized by EGFRab $^{\mathrm{A} 647}$, and the EGFRab ${ }^{\mathrm{A} 647} \mathrm{BF}$ represents the percentile of EGFRab ${ }^{\mathrm{A} 647}$ binding to EGFR $^{\mathrm{GFP}}$. 

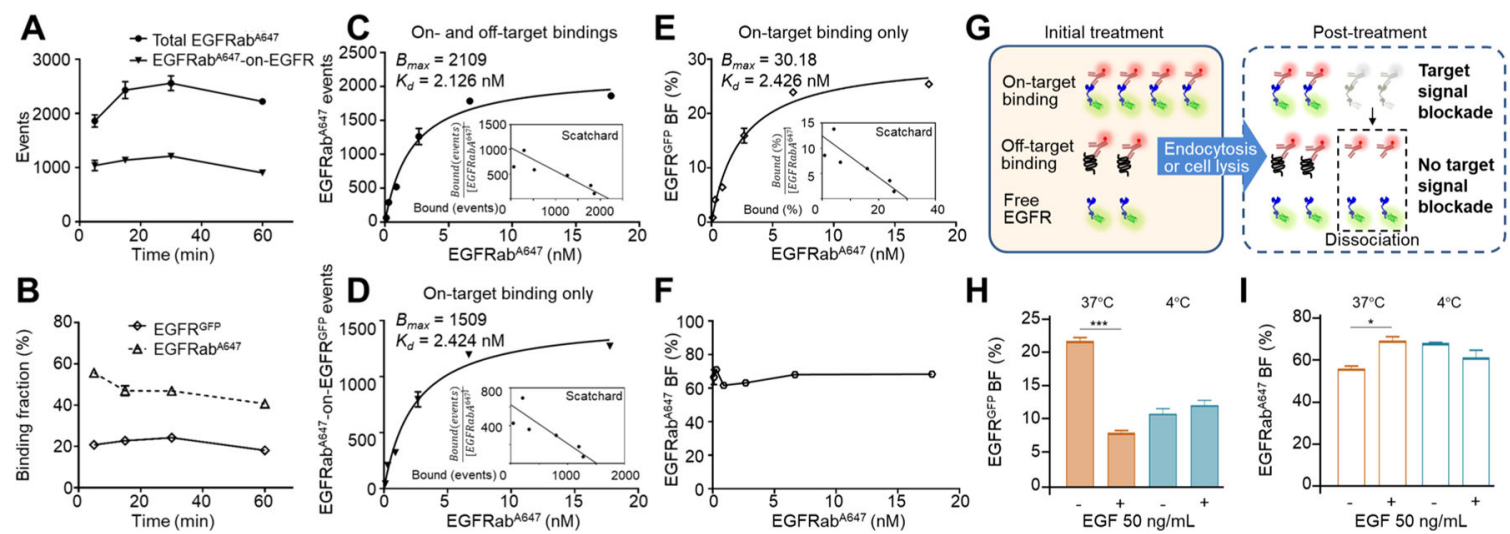

Figure 2.

Digital quantification of EGFR-EGFRab binding in CHO-EGFR GFP cells. (A) Numbers of EGFRab $^{\mathrm{A} 647}$-EGFR ${ }^{\mathrm{GFP}}$ events and total EGFRab ${ }^{\mathrm{A} 647}$ events after 5-60 min of EGFRab $^{\mathrm{A} 647}$ treatment. These EGFRab ${ }^{\mathrm{A} 647}$ events were associated with CHO-EGFR GFP cells after PBS wash. (B) Binding fractions of EGFR ${ }^{\mathrm{GFP}}$ and EGFRab ${ }^{\mathrm{A} 647}$ after treatments at different times. (C) Total EGFRab ${ }^{\mathrm{A} 647}$ events following treatment with different concentrations of EGFRab ${ }^{\mathrm{A} 647}$ are plotted, and the events include on- and off-target binding. (D) Receptor occupied EGFRab ${ }^{\mathrm{A} 647}$ events are plotted, and the bound events only include on-target binding. (E) Binding saturation curve was plotted on the basis of the EGFR GFP binding fraction following treatment with different concentrations of EGFRab ${ }^{\mathrm{A} 647}$. In (CE), the Scatchard plots were used to determine the dissociation constants $\left(K_{\mathrm{d}}\right)$ and a maximum number of targetable EGFRs $\left(B_{\max }\right)$. (F) EGFRab ${ }^{\mathrm{A} 647}$ binding fraction involved in EGFRab ${ }^{\text {A647-EGFR }}{ }^{\text {GFP }}$ events. Each data point was determined based on 5,000 EGFR ${ }^{\text {GFP }}$ events counted, $n=3$. (G) Coincident detection method is able to dissect the amount of antibody effectively blocking the target. (H) CHO-EGFR GFP cells were treated with or without $50 \mathrm{ng} / \mathrm{mL}$ EGF for $30 \mathrm{~min}$ at 37 or $4{ }^{\circ} \mathrm{C}$, respectively. The cells were later treated with $15 \mathrm{nM}$ EGFRab ${ }^{\mathrm{A} 647}$ and incubated for $5 \mathrm{~min}$. After incubation, the cells were washed with PBS and lysed for DRO analysis. The results of EGFR GFP binding fraction show that the EGF-induced endocytosis reduced the number of EGFR on the plasma membrane, so the binding of antibodies to EGFRs also decreased. In contrast, the low temperature suppressed EGFR endocytosis, and there is no significant difference between groups treated with or without EGF. (I) EGFRab ${ }^{\mathrm{A} 647}$ binding fractions with or without EGF stimulation at 37 or $4{ }^{\circ} \mathrm{C}$. Each data point was determined based on 5,000 EGFR GFP events counted with sample number $n=3$. Statistical comparison is performed using paired $t$ test, where the asterisk represents statistical significance: $* * * p<0.001$ and $* p<0.05$. The error bars represent standard deviations. 


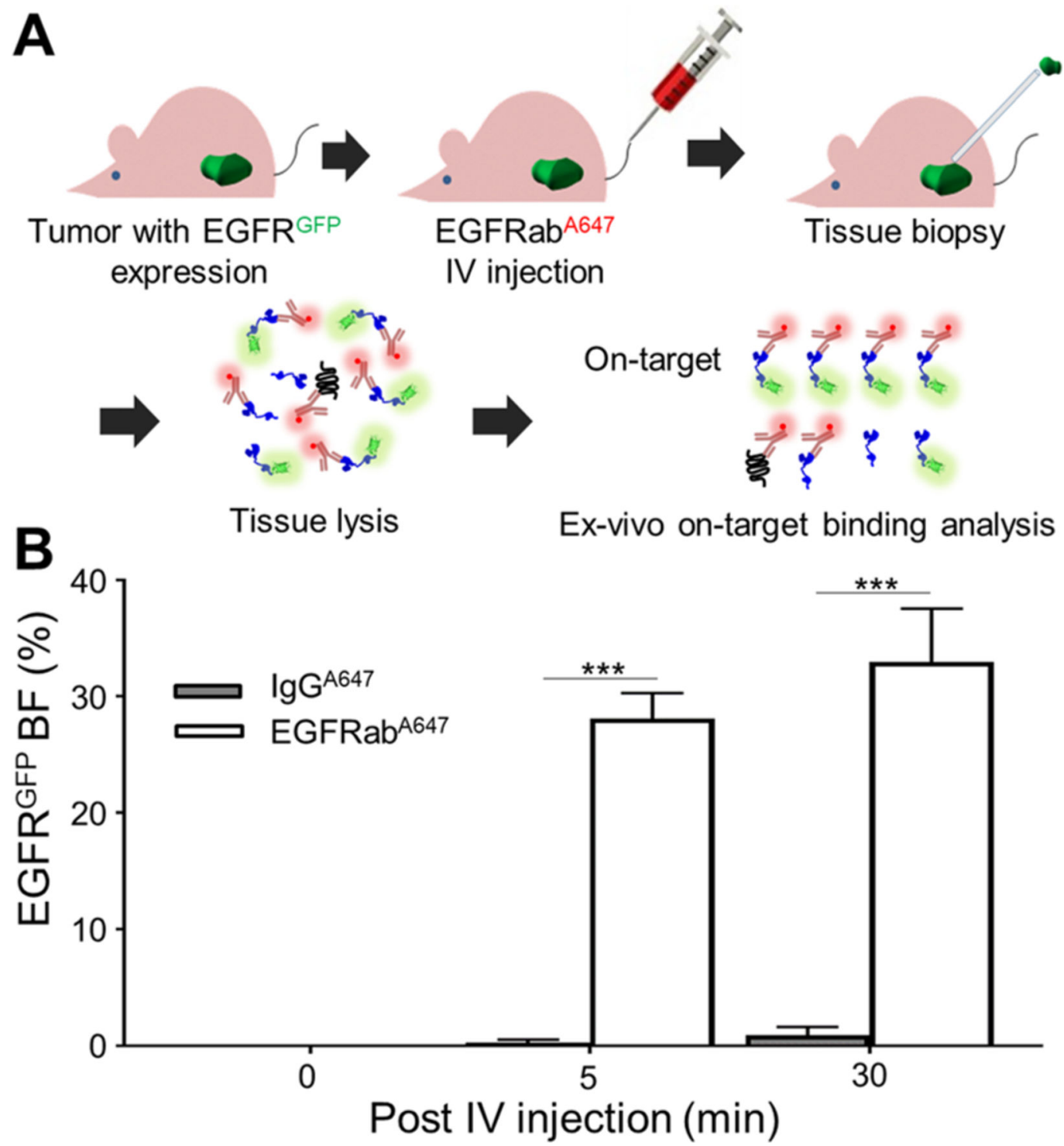

Figure 3.

Quantification of the EGFRab ${ }^{\mathrm{A} 647}$ binding fraction in the tumor xenograft model. (A) Mice with subcutaneous GEO-EGFR ${ }^{\text {GFP }}$ tumors were treated with intravenous injection (IV) of $100 \mu$ EGFRab $^{\mathrm{A} 647}$ or isotype control $\mathrm{IgG}^{\mathrm{A} 647}$. Small tumor samples were collected at injection and 5 and 30 min after injection and lysed for DRO analysis. (B) EGFR GFP binding fraction in xenograft tumor tissue. Each data point was determined based on 5,000 overall EGFR ${ }^{\mathrm{GFP}}$ events counted, $n=3$. Statistical comparison is performed using paired $t$ test, where the asterisk represents statistical significance: $* * * p<0.001$. The error bars represent standard deviations. 